\title{
Persistent current in a chain of two Holstein-Hubbard rings in the presence of Rashba spin-orbit interaction
}

\author{
A. Chatterjee ${ }^{2}$, M. O. Smolkina ${ }^{1}$, I. Y. Popov ${ }^{1}$ \\ ${ }^{1}$ ITMO University, Kronverkskiy, 49, St. Petersburg, 197101, Russia \\ ${ }^{2}$ University of Hyderabad, Hyderabad-500046, India \\ acsp@uohyd.ernet.in, vega14@mail.ru, popov1955@gmail.com
}

DOI 10.17586/2220-8054-2019-10-1-50-62

\begin{abstract}
The persistent current in a chain of two quantum rings threaded by an Aharonov-Bohm flux is studied in the presence of electron-phonon interactions and Rashba spin-orbit coupling. The chain is modeled by the Holstein-Hubbard-Rashba Hamiltonian, the phonon's degrees of freedom were eliminated by the conventional Lang-Firsov transformation, the effective electronic Hamiltonian was diagonalized by using the Hartree-Fock approximation. The equations for ground state energy, persistent current and Drude weight were also obtained. The persistent current was calculated by differentiating the GS energy. The dependence of ground state energy, persistent current and Drude weight as a functions of flux for different values of Rashba spin-orbit interaction was numerically shown. The effects of Aharonov-Bohm flux, temperature, chemical potential spin-orbit interaction and electron-phonon interaction on the persistent current were also investigated.
\end{abstract}

Keywords: spin-orbit interaction, persistent current.

Received: 2 January 2019

Revised: 10 February 2019

\section{Introduction}

A normal metal ring threaded by a magnetic flux can sustain a loss-less current, commonly referred to as the persistent current, because of the quantum mechanical phase coherence effect. This novel effect was first predicted by Buttiker, Imry and Landauer [1] and later quite a few other aspects of this problem were reported by several other investigators [2]. For example, it was proposed that the persistent current would be periodic in flux. Several experiments [3] have established quite unequivocally that persistent current can exist in a semiconductor quantum ring and it is indeed periodic in nature. The Hubbard model turns out to be a suitable model to investigate the persistent current in a quantum ring consisting of discrete sites [4]. The effects of spin-orbit interactions [5] are also found to be pronounced in quantum rings. Recently the effect of electron-phonon interaction on the persistent current in a correlated quantum ring in the presence of Rashba spin-orbit interaction has been studied by Monisha et al. [6] using the one-dimensional Holstein-Hubbard model. Using of quantum rings is rather popular in nanoscience (see, e.g., [7,8]. The advantage with the Rashba interaction is that it can be manipulated by tuning the external magnetic field and concomitantly the persistent current can be controlled. This is one of the main principles on which spintronics is based.

In the present work, we study the effect of Rashba spin-orbit interaction on persistent current in a chain of two Holstein-Hubbard rings threaded by an Aharonov-Bohm flux. We consider the magnetic flux going through the quantum rings in such a way that the magnetic field is zero at the radii of the rings. It is a quantum mechanical phenomenon which can be observed in small metallic rings whose size is comparable to the electron coherence length. The energy spectrum is periodic in flux and consequently, the persistent current which is the change in ground state energy with respect to the magnetic flux is also periodic in flux.

\section{Model of two Holstein-Hubbard rings in the presence of Rashba spin-orbit interaction}

The chain of two Holstein-Hubbard rings considered in this work is shown in Fig. 1. Both of the rings consist of discrete lattice sites and the electrons can hop from one site to another.

The Hamiltonian for a chain of two Holstein-Hubbard rings threaded by a magnetic flux is written in the presence of Rashba spin-orbit interaction as:

$$
H=H_{e l}+H_{p}+H_{e p}+H_{s o}
$$

with

$$
H_{e l}=\varepsilon_{0} \sum_{i} c_{i}^{+} c_{i}+\varepsilon_{0} \sum_{k} c_{k}^{+} c_{k}-t e^{\gamma \theta} \sum_{\langle i, j\rangle}\left(c_{i}^{+} c_{j}+h . c\right)-\frac{t}{2} e^{\gamma \theta} \sum_{\langle k, l\rangle}\left(c_{k}^{+} c_{l}+h . c\right)+U \sum_{i} n_{i \uparrow} n_{i \downarrow}+U \sum_{k} n_{k \uparrow} n_{k \downarrow},
$$




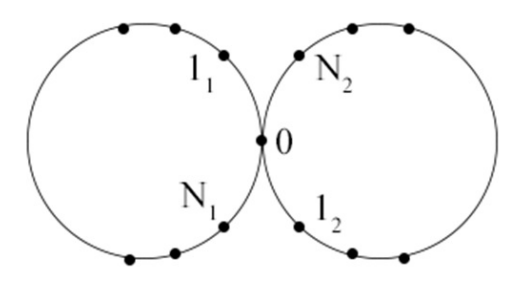

FIG. 1. Chain of two Holstein-Hubbard rings. The intersection point is 0 , points $1_{1}, N_{1}, 1_{2}, N_{2}$ are its nearest neighbors

$$
\begin{gathered}
H_{p}=\hbar \omega_{0} \sum_{i}\left(b_{i}^{+} b_{i}+\frac{1}{2}\right)+\hbar \omega_{0} \sum_{k}\left(b_{k}^{+} b_{k}+\frac{1}{2}\right), \\
H_{e p}=g_{1} \sum_{i} n_{i}\left(b_{i}+b_{i}^{+}\right)+g_{1} \sum_{k} n_{k}\left(b_{k}+b_{k}^{+}\right)+g_{2} \sum_{\langle i, j\rangle} n_{i}\left(b_{j}+b_{j}^{+}\right)+\frac{g_{2}}{2} \sum_{\langle k, l\rangle} n_{k}\left(b_{l}+b_{l}^{+}\right), \\
H_{s o}=-t \sum_{\langle i, j\rangle}\left(c_{i}^{+} t_{s o} e^{\gamma \theta} c_{j}+h . c\right)-\frac{t}{2} \sum_{\langle k, l\rangle}\left(c_{k}^{+} t_{s o} e^{\gamma \theta} c_{l}+h . c\right),
\end{gathered}
$$

where $\langle i, j\rangle$ is any pair of two nearest neighbors on the ring, except at the point of intersection and its nearest neighbors. Pairs $\langle k, l\rangle$ include the point of intersection and its nearest neighbors. $H_{e l}$, given by Eq. (2), is the electronic Hamiltonian which consists of six terms. The first two terms stand for the site energies of the two rings, $\varepsilon_{0}$ referring to the on-site energy, $c_{i}=\left(\begin{array}{c}c_{i \uparrow} \\ c_{i \downarrow}\end{array}\right), c_{i \sigma}^{\dagger}\left(c_{i \sigma}\right)$ denoting the creation (annihilation) operator for an electron at site $i$ with spin $\sigma, i$ taking values $1,2,3,4, \ldots N$, where $N$ is the total number of sites in each ring except the point of intersection and its nearest neighbors, $k$ is the number of point of intersection and its nearest neighbors (points $0,1_{1}, 1_{2}, N_{1}, N_{2}$ on Fig. 1). Next two terms describe the kinetic energies, $t$ being the hopping integral and $\theta=(2 \pi \Phi / n)$ the Aharonov-Bohm phase arising from the quantized magnetic flux $\Phi=m \Phi_{0}$ where $m$ is an integer and $\Phi_{0}=h c / e$ is the elementary flux quantum. The last two terms represent the onsite Coulomb repulsion with $U$ measuring the strength of the repulsion and $n_{i \sigma}=c_{i \sigma}^{\dagger} c_{i \sigma}$ refers to the operator corresponding to the electron number at site $i$ with spin $\sigma . H_{p}$, given by Eq. (3), is the sum of the Hamiltonians for non-interacting phonons in the two rings, $b_{i}^{\dagger}\left(b_{i}\right)$ being the phonon creation (annihilation) operator at site $i$ and $\omega_{0}$ the phonon frequency which is assumed to be dispersionless. $H_{e p}$, given by Eq. (4), describes the on-site and nearest-neighbor electron-phonon interactions with $g_{1}, g_{2}$ denoting the corresponding coupling strengths. We assume that $g_{2}$ is smaller than $g_{1}$ by an order of magnitude or so. Finally, Eq. (5) represents the Rashba spin-orbit interaction with $t_{s o}$ as the spin-orbit coupling constant given by: $t_{s o}=i \alpha\left(\sigma_{x} \cos \varphi_{i j}+\sigma_{y} \sin \varphi_{i j}\right)$ where $\sigma_{m}$ is the $m$-th component of the Pauli matrix $\sigma, \varphi_{i j}=\left(\varphi_{i}+\varphi_{j}\right) / 2$ with $\varphi_{i}=2 \pi(i-1) / N, i$ being the site index along the azimuthal direction of the ring.

\section{Analytical results}

To eliminate the phonons we carry out the celebrated Lang-Firsov transformation [7,9] on the Hamiltonian (1) with the generator:

$$
R=\frac{1}{\hbar \omega_{0}}\left(g_{1} \sum_{i \sigma} n_{i \sigma}\left(b_{i}^{+}-b_{i}\right)+g_{1} \sum_{k \sigma} n_{k \sigma}\left(b_{k}^{+}-b_{k}\right)+g_{2} \sum_{\langle i, j\rangle \sigma} n_{i \sigma}\left(b_{j}^{+}-b_{j}\right)+\frac{g_{2}}{2} \sum_{\langle k, l\rangle \sigma} n_{k \sigma}\left(b_{l}^{+}-b_{l}\right)\right)
$$


The transformed Hamiltonian is given by:

$$
\begin{gathered}
\tilde{H}=e^{R} H e^{-R}=H+[R, H]+\frac{1}{2 !}[R,[R, H]]+\ldots= \\
-\frac{1}{\hbar \omega_{0}}\left(g_{1}^{2}+z g_{2}^{2}\right) \sum_{i \sigma} n_{i \sigma}-t e^{\iota \theta} \sum_{\langle i, j\rangle \sigma} e^{\left(Y_{i}-Y_{j}\right)} c_{i \sigma}^{+} c_{j \sigma}-\iota e^{\iota \theta} \sum_{\langle i, j\rangle \sigma}\left[\begin{array}{cc}
0 & P \\
Q & 0
\end{array}\right] e^{\left(Y_{i}-Y_{j}\right)} c_{i \sigma}^{+} c_{j \sigma}+ \\
\left.+\left(U-\frac{2}{\hbar \omega_{0}}\left(g_{1}^{2}+z g_{2}^{2}\right)\right) \sum_{i} n_{i \uparrow} n_{i \downarrow}-\frac{g_{2}^{2}}{\hbar \omega_{0}} \sum_{i \Delta \sigma \sigma^{\prime}} n_{i \sigma} n_{i+\Delta \sigma^{\prime}}-\frac{2}{\hbar \omega_{0}} g_{2} g_{1}\right) \sum_{\langle i, j\rangle \sigma \sigma^{\prime}} n_{i \sigma} n_{j \sigma^{\prime}}+\hbar \omega_{0} \sum_{i}\left(b_{i}^{+} b_{i}+\frac{1}{2}\right)- \\
-\frac{1}{\hbar \omega_{0}} \sum_{k \sigma}\left(g_{1}^{2}+\frac{z_{k \sigma}}{4} g_{2}^{2}\right) n_{k \sigma}-\frac{t}{2} e^{\iota \theta} \sum_{\langle k, l\rangle \sigma} e^{\left(Y_{k}-Y_{l}\right)} c_{k \sigma}^{+} c_{l \sigma}-\frac{\iota}{2} e^{\iota \theta} \sum_{\langle k, l\rangle}\left[\begin{array}{cc}
0 & P \\
Q & 0
\end{array}\right] e^{\left(Y_{k}-Y_{l}\right)} c_{k}^{+} c_{l}+ \\
+\sum_{k}\left(U-\frac{2}{\hbar \omega_{0}}\left(g_{1}^{2}+\frac{z_{1}}{4} g_{2}^{2}\right)\right) n_{k \uparrow} n_{k \downarrow}-\frac{g_{2}^{2}}{2 \hbar \omega_{0}} \sum_{\langle k, l\rangle \sigma \sigma^{\prime}, k, l \neq 0} n_{k \sigma} n_{l \sigma^{\prime}}+\frac{1}{\hbar \omega_{0}} g_{2} g_{1} \sum_{\langle k, l\rangle \sigma \sigma^{\prime}} n_{k \sigma} n_{l \sigma^{\prime}}+ \\
+\hbar \omega_{0} \sum_{k}\left(b_{k}^{+} b_{k}+\frac{1}{2}\right) .
\end{gathered}
$$

The effective electronic Hamiltonian can be obtained by taking the zero-phonon averaging:

$$
H_{\text {eff }}=\left\langle 0\left|e^{R} H e^{-R}\right| 0\right\rangle=\langle 0|\tilde{H}| 0\rangle .
$$

It is easy to see that $\left[Y_{i}, Y_{j}\right]=0,\left[Y_{k}, Y_{l}\right]=0$ and

$$
\left\langle 0\left|e^{Y_{i}-Y_{j}}\right| 0\right\rangle=e^{-\left(\frac{1}{\hbar \omega_{0}}\right)^{2}\left[\left(g_{1}-g_{2}\right)^{2}+(z-1) g_{2}^{2}\right]}, \quad\left\langle 0\left|e^{Y_{k}-Y_{l}}\right| 0\right\rangle=e^{-\left(\frac{1}{\hbar \omega_{0}}\right)^{2}\left[\left(g_{1}-g_{2}\right)^{2}+\left(z_{k}-1\right) g_{2}^{2}\right]} .
$$

Using these results, we can write the effective electronic Hamiltonian in a simplified form as:

$$
\begin{aligned}
& H_{e f f}=\epsilon_{0}^{e} \sum_{i \sigma} n_{i \sigma}-t_{e} e^{\iota \theta} \sum_{\langle i, j\rangle \sigma} c_{i \sigma}^{+} c_{j \sigma}-t_{S O-e}^{i j} e^{\iota \theta} \sum_{\langle i, j\rangle \sigma} c_{i \sigma}^{+} c_{j \sigma}+U_{e} \sum_{i} n_{i \uparrow} n_{i \downarrow}+ \\
& +\sum_{k \sigma} \epsilon_{0 k}^{e} n_{k \sigma}-\frac{e^{\iota \theta}}{2} \sum_{\langle k, l\rangle \sigma} t_{e k} c_{k \sigma}^{+} c_{l \sigma}-\frac{e^{\iota \theta}}{2} \sum_{\langle k, l\rangle \sigma} t_{S O-e k}^{k l} c_{k \sigma}^{+} c_{l \sigma}+\sum_{k} U_{e k} n_{k \uparrow} n_{k \downarrow}
\end{aligned}
$$

where $\epsilon_{0}^{e}=-\frac{\left(g_{1}^{2}+z g_{2}^{2}\right)}{\hbar \omega_{0}}, z$ is the number of nearest neighbors (for all the sites except the point of intersection and its nearest neighbors), $\epsilon_{0 k}^{e}=-\frac{\left(g_{1}^{2}+\frac{z_{k}}{4} g_{2}^{2}\right)}{\hbar \omega_{0}}, z_{k}$ - number of nearest neighbors (for the point of intersection and its nearest neighbors),

$$
\begin{array}{cl}
t_{e}=t e^{-\left(\frac{1}{\hbar \omega_{0}}\right)^{2}\left[\left(g_{1}-g_{2}\right)^{2}+(z-1) g_{2}^{2}\right]}, & t_{e k}=t e^{-\left(\frac{1}{\hbar \omega_{0}}\right)^{2}\left[\left(g_{1}-g_{2}\right)^{2}+\left(z_{k}-1\right) g_{2}^{2}\right]}, \\
t_{S O-e}^{i j}=\iota \mathrm{A} e^{-\left(\frac{1}{\hbar \omega_{0}}\right)^{2}\left[\left(g_{1}-g_{2}\right)^{2}+(z-1) g_{2}^{2}\right]}, & t_{S O-e k}^{i j}=\iota \mathrm{A}_{k} e^{-\left(\frac{1}{\hbar \omega_{0}}\right)^{2}\left[\left(g_{1}-g_{2}\right)^{2}+\left(z_{k}-1\right) g_{2}^{2}\right]}, \\
\mathrm{A}=\left[\begin{array}{ll}
0 & P \\
Q & 0
\end{array}\right]=\left[\begin{array}{ll}
0 & \alpha e^{-\iota \phi_{i j}} \\
\alpha e^{\iota \phi_{i j}} & 0
\end{array}\right], & \mathrm{A}_{k}=\left[\begin{array}{ll}
0 & P \\
Q & 0
\end{array}\right]=\left[\begin{array}{ll}
0 & \alpha e^{-\iota \phi_{k l}} \\
\alpha e^{\iota \phi_{k l}} & 0
\end{array}\right], \\
U_{e}=U-\frac{2}{\hbar \omega_{0}}\left(g_{1}^{2}+z g_{2}^{2}\right), & U_{e k}=U-\frac{2}{\hbar \omega_{0}}\left(g_{1}^{2}+\frac{z_{k}}{4} g_{2}^{2}\right) .
\end{array}
$$

Next, we perform the unitary transformation on the effective Hamiltonian $H_{\text {eff }}$ with the matrix:

$$
U_{m}=\frac{1}{\sqrt{2}}\left[\begin{array}{ll}
1 & -1 \\
e^{\frac{2 \pi \iota}{N}\left(m-\frac{1}{2}\right)} & e^{\frac{2 \pi \iota}{N}\left(m-\frac{1}{2}\right)}
\end{array}\right] .
$$

This transformation helps us to eliminate the index dependence of the spin-orbit interaction coefficients $t_{S O-e}^{i j}$, $t_{S O-e k}^{i j}$ and transforms the old operators $c_{i}, c_{k}$ to a new set of operators $\underset{i}{\underset{c}{c}}=U_{i}^{+} c_{i}, \underset{k}{\underset{c}{c}}=U_{k}^{+} c_{k}$. The transformed effective Hamiltonian is given by: 


$$
\begin{aligned}
& H_{e}=\epsilon_{0}^{e} \sum_{i \sigma} \tilde{n}-\frac{1}{2} e^{\iota \theta} \sum_{\langle i, j\rangle \sigma} c_{i \sigma}^{+}\left[t_{e}+\iota \alpha_{e}\right] \mathrm{B} c_{j \sigma}+U_{e} \sum_{i} \underset{i \uparrow}{\tilde{n}} \tilde{\sim} \underset{i \downarrow}{\tilde{n}}+\sum_{k \sigma} \epsilon_{0 k}^{e} \underset{k \sigma}{\tilde{n}}-\frac{1}{4} e^{\iota \theta} \sum_{\langle k, l\rangle \sigma} c_{k \sigma}^{+}\left[t_{e k}+\iota \alpha_{e k}\right] B c_{l \sigma}+
\end{aligned}
$$

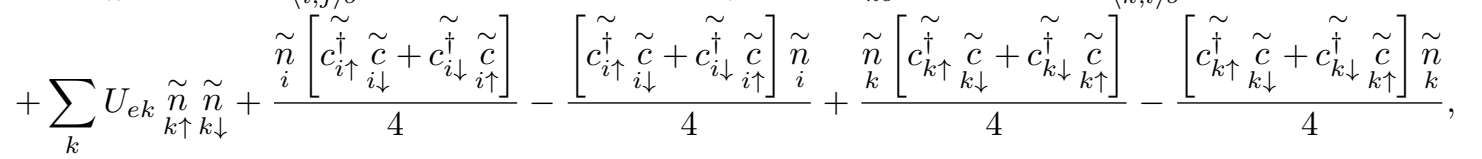

where

$$
\begin{gathered}
\alpha_{e}=\alpha e^{-\left(\frac{1}{\hbar \omega_{0}}\right)^{2}\left[\left(g_{1}-g_{2}\right)^{2}+(z-1) g_{2}^{2}\right]}, \quad \alpha_{e k}=\alpha e^{-\left(\frac{1}{\hbar \omega_{0}}\right)^{2}\left[\left(g_{1}-g_{2}\right)^{2}+\left(z_{k}-1\right) g_{2}^{2}\right]}, \\
\mathrm{B}=\left[\begin{array}{cc}
1+e^{\frac{2 \pi \iota}{N}} & -1+e^{\frac{2 \pi \iota}{N}} \\
-1+e^{\frac{2 \pi \iota}{N}} & 1+e^{\frac{2 \pi \iota}{N}}
\end{array}\right] .
\end{gathered}
$$

Next, we use the mean-field approximation to linearize the quadratic terms in $H_{e}$ (see equation (9). In 1D systems, for a half filled band the electron-phonon interaction can cause the distortion of the lattice leading to dimerization with the unit cell getting doubled.

To proceed further, we divide the lattice into two sublattices: even numbered sites-A,and odd numbered sites-B. Using some algebraic simplifications following Cabib and Callen [10] we obtain:

$$
\begin{aligned}
& H_{e}^{m}=\sum_{i=1}^{N} \tilde{c}_{i}^{+}\left[C+(-1)^{i} D\right] \tilde{c}_{i}-e^{\iota\left(\theta+\frac{\pi}{N}\right)} \sum_{<i j>\sigma, i, j \neq 0}^{N} \tilde{c}_{i \sigma}^{+}\left[t_{e} E+\iota \alpha_{e} F\right] \tilde{c}_{j \sigma}+K_{1}+ \\
& \sum_{k=0}^{N} \tilde{c}_{k}^{+}\left[C_{k}+(-1)^{k} D_{k}\right] \tilde{c}_{k}-e^{\iota\left(\theta+\frac{\pi}{N}\right)} \sum_{<k l>\sigma}^{N} \tilde{c}_{k \sigma}^{+}\left[t_{e k} E+\iota \alpha_{e k} F\right] \tilde{c}_{l \sigma}+K_{2} .
\end{aligned}
$$

where

$$
\begin{aligned}
& C=\left[\begin{array}{ll}
\varepsilon_{A B \uparrow}^{+} & 0 \\
0 & \varepsilon_{A B \downarrow}^{+}
\end{array}\right], \quad C_{k}=\left[\begin{array}{ll}
\varepsilon_{A B \uparrow k}^{+} & 0 \\
0 & \varepsilon_{A B \downarrow k}^{+}
\end{array}\right], \\
& \varepsilon_{A B \uparrow}^{+}=\frac{\varepsilon_{A \uparrow}+\varepsilon_{B \uparrow}}{2}, \quad \varepsilon_{A B \downarrow}^{+}=\frac{\varepsilon_{A \downarrow}+\varepsilon_{B \downarrow}}{2}, \quad \varepsilon_{A B \uparrow k}^{+}=\frac{\varepsilon_{A \uparrow k}+\varepsilon_{B \uparrow k}}{2}, \quad \varepsilon_{A B \downarrow k}^{+}=\frac{\varepsilon_{A \downarrow k}+\varepsilon_{B \downarrow k}}{2}, \\
& D=\left[\begin{array}{ll}
\varepsilon_{A B \uparrow}^{-} & 0 \\
0 & \varepsilon_{A B \downarrow}^{-}
\end{array}\right], \quad D_{k}=\left[\begin{array}{ll}
\varepsilon_{A B \uparrow k}^{-} & 0 \\
0 & \varepsilon_{A B \downarrow k}^{-}
\end{array}\right], \\
& \varepsilon_{A B \uparrow}^{-}=\frac{\varepsilon_{A \uparrow}-\varepsilon_{B \uparrow}}{2}, \quad \varepsilon_{A B \downarrow}^{-}=\frac{\varepsilon_{A \downarrow}-\varepsilon_{B \downarrow}}{2}, \quad \varepsilon_{A B \uparrow k}^{-}=\frac{\varepsilon_{A \uparrow k}-\varepsilon_{B \uparrow k}}{2}, \quad \varepsilon_{A B \downarrow k}^{-}=\frac{\varepsilon_{A \downarrow k}-\varepsilon_{B \downarrow k}}{2}, \\
& \varepsilon_{A \uparrow}=\varepsilon_{0}^{e}+\frac{U_{e}(c-s)}{2}, \quad \varepsilon_{B \uparrow}=\varepsilon_{0}^{e}-\frac{U_{e}(c-s)}{2}, \quad \varepsilon_{A \downarrow}=\varepsilon_{0}^{e}+\frac{U_{e}(c+s)}{2}, \quad \varepsilon_{B \downarrow}=\varepsilon_{0}^{e}-\frac{U_{e}(c+s)}{2}, \\
& \varepsilon_{A \uparrow k}=\varepsilon_{0 k}^{e}+\frac{U_{e k}(c-s)}{2}, \quad \varepsilon_{B \uparrow k}=\varepsilon_{0 k}^{e}-\frac{U_{e k}(c-s)}{2}, \quad \varepsilon_{A \downarrow k}=\varepsilon_{0 k}^{e}+\frac{U_{e k}(c+s)}{2}, \quad \varepsilon_{B \downarrow k}=\varepsilon_{0 k}^{e}-\frac{U_{e k}(c+s)}{2}, \\
& E=\left[\begin{array}{cc}
\cos \left(\frac{\pi}{N}\right) & \iota \sin \left(\frac{\pi}{N}\right) \\
\iota \sin \left(\frac{\pi}{N}\right) & \cos \left(\frac{\pi}{N}\right)
\end{array}\right], \quad F=\left[\begin{array}{cc}
\cos \left(\frac{\pi}{N}\right) & \iota \sin \left(\frac{\pi}{N}\right) \\
-\iota \sin \left(\frac{\pi}{N}\right) & -\cos \left(\frac{\pi}{N}\right)
\end{array}\right] \\
& K_{1}=\frac{N U_{e}\left(n^{2}-c^{2}+s^{2}\right)}{4}, \quad K_{2}=\frac{N U_{e k}\left(n^{2}-c^{2}+s^{2}\right)}{4}, \\
& n=\frac{\left[\left(n_{A \uparrow}+n_{A \downarrow}\right)+\left(n_{B \uparrow}+n_{B \downarrow}\right)\right]}{2} \text {, } \\
& c=\frac{\left[\left(n_{A \uparrow}+n_{A \downarrow}\right)-\left(n_{B \uparrow}+n_{B \downarrow}\right)\right]}{2}, \\
& s=\frac{\left[\left(n_{A \uparrow}-n_{A \downarrow}\right)-\left(n_{B \uparrow}-n_{B \downarrow}\right)\right]}{2},
\end{aligned}
$$

where $n$ is the electron concentration, $c$ is the charge density wave order parameter, and $s$ is the spin density wave order parameter. Performing the Fourier transformation:

$$
\tilde{c}_{m \sigma}=\frac{1}{\sqrt{N}} \sum_{\xi} e^{\iota \xi m a} \tilde{c}_{\xi \sigma},
$$

where $a$ is the lattice spacing and making the redefinitions: $\tilde{c}_{i \sigma}\left(\tilde{c}_{i \sigma}^{+}\right)$as $c_{i \sigma}\left(c_{i \sigma}^{+}\right)$and $\tilde{c}_{k \sigma}\left(\tilde{c}_{k \sigma}^{+}\right)$as $c_{k \sigma}\left(c_{k \sigma}^{+}\right)$and using the following identities: 
$\sum_{m=1}^{\frac{N}{2}} c_{2 m}^{+} c_{2 m}=\frac{1}{2} \sum_{\xi} c_{\xi}^{+} c_{\xi}+\frac{1}{2} \sum_{\xi} c_{\xi}^{+} c_{\xi+\frac{\pi}{a}}$, for even sites,

$\sum_{m=1}^{\frac{N}{2}} c_{2 m-1}^{+} c_{2 m-1}=\frac{1}{2} \sum_{\xi} c_{\xi}^{+} c_{\xi}-\frac{1}{2} \sum_{\xi} c_{\xi}^{+} c_{\xi+\frac{\pi}{a}}$, for odd sites, we can separate the Hamiltonian into even and odd sited terms to get the effective mean-field Hamiltonian as:

$$
H_{e}^{M}=2 \sum_{\xi=-\pi / a, \xi \neq 0}^{\pi / a} c_{\xi}^{+} G c_{\xi}+2 \sum_{\xi=-\pi / a, \xi \neq 0, \xi+(\pi / a) \neq 0}^{\pi / a} c_{\xi}^{+} D c_{\xi+(\pi / a)}+c_{0}^{+} G_{0} c_{0}+c_{0}^{+} D_{0} c_{\pi / a}+2 K_{1}+K_{0},
$$

where

$$
\begin{gathered}
G=\left[\begin{array}{ll}
\varepsilon_{A B \uparrow}^{+}+\alpha_{11} & \alpha_{12} \\
\alpha_{21} & \varepsilon_{A B \downarrow}^{+}+\alpha_{22}
\end{array}\right], \quad D=\left[\begin{array}{ll}
\varepsilon_{A B \uparrow}^{-} & 0 \\
0 & \varepsilon_{A B \downarrow}^{-}
\end{array}\right], \\
G_{0}=\left[\begin{array}{ll}
\varepsilon_{A B \uparrow}^{-}-\varepsilon_{A B \uparrow}^{+}+\frac{1}{2}\left[\varepsilon_{A B \uparrow 0}^{+}-\varepsilon_{A B \uparrow 0}^{-}\right]+\alpha_{11,0} & \alpha_{12,0} \\
\alpha_{21,0} & \varepsilon_{A B \downarrow}^{-}-\varepsilon_{A B \downarrow}^{+}+\frac{1}{2}\left[\varepsilon_{A B \downarrow 0}^{+}-\varepsilon_{A B \downarrow 0}^{-}\right]+\alpha_{22,0}
\end{array}\right], \\
D_{0}=\left[\begin{array}{ll}
\varepsilon_{A B \uparrow}^{+}-\varepsilon_{A B \uparrow}^{-}-\frac{1}{2}\left[\varepsilon_{A B \uparrow 0}^{+}-\varepsilon_{A B \uparrow 0}^{-}\right] & 0 \\
0 & \varepsilon_{A B \downarrow}^{+}-\varepsilon_{A B \downarrow}^{-}-\frac{1}{2}\left[\varepsilon_{A B \downarrow 0}^{+}-\varepsilon_{A B \downarrow 0}^{-}\right]
\end{array}\right], \\
\alpha_{11}=-2 t_{e} \cos (\pi / N) \cos (\xi a+\theta+\pi / N)+2 \alpha_{e} \cos (\pi / N) \sin (\xi a+\theta+\pi / N), \\
\alpha_{12}=2 t_{e} \sin (\pi / N) \sin (\xi a+\theta+\pi / N)+2 \alpha_{e} \sin (\pi / N) \cos (\xi a+\theta+\pi / N), \\
\alpha_{21}=2 t_{e} \sin (\pi / N) \sin (\xi a+\theta+\pi / N)-2 \alpha_{e} \sin (\pi / N) \cos (\xi a+\theta+\pi / N), \\
\alpha_{22}=-2 t_{e} \cos (\pi / N) \cos (\xi a+\theta+\pi / N)-2 \alpha_{e} \cos (\pi / N) \sin (\xi a+\theta+\pi / N), \\
\alpha_{11,0}=2\left[2 t_{e}-t_{e 0}\right] \cos (\pi / N) \cos (\theta+\pi / N)-2\left[2 \alpha_{e}+\alpha_{e 0}\right] \cos (\pi / N) \sin (\theta+\pi / N), \\
\alpha_{12,0}=2\left[-2 t_{e}+t_{e 0}\right] \sin (\pi / N) \sin (\theta+\pi / N)-2\left[2 \alpha_{e}-\alpha_{e 0}\right] \sin (\pi / N) \cos (\theta+\pi / N), \\
\alpha_{21,0}=2\left[-2 t_{e}+t_{e 0}\right] \sin (\pi / N) \sin (\theta+\pi / N)-2\left[-2 \alpha_{e}+\alpha_{e 0}\right] \sin (\pi / N) \cos (\theta+\pi / N), \\
\alpha_{22,0}=2\left[2 t_{e}-t_{e 0}\right] \cos (\pi / N) \cos (\theta+\pi / N)+2\left[+2 \alpha_{e}+\alpha_{e 0}\right] \cos (\pi / N) \sin (\theta+\pi / N) .
\end{gathered}
$$

It is convenient to work in the reduced zone scheme and so we choose the domain of $\xi$ as: $-\pi / 2 a$. The matrix elements then satisfy the equation: $\alpha_{i j}(\xi+\pi / a)=-\alpha_{i j}(\xi)$. The effective Hamiltonian is finally given in the mean-field approximation by:

$$
H_{e}^{M}=2 \sum_{\xi=0}^{\pi}\left(c_{k \uparrow}^{+} c_{k \downarrow}^{+} c_{k+\pi, \uparrow}^{+} c_{k+\pi, \downarrow}^{+}\right) W\left(\begin{array}{l}
c_{k \uparrow} \\
c_{k \downarrow} \\
c_{k+\pi, \uparrow} \\
c_{k+\pi, \downarrow}
\end{array}\right)+\left(c_{0 \uparrow}^{+} c_{0 \downarrow}^{+} c_{\pi, \uparrow}^{+} c_{\pi, \downarrow}^{+}\right) W_{0}\left(\begin{array}{c}
c_{0 \uparrow} \\
c_{0 \downarrow} \\
c_{\pi, \uparrow} \\
c_{\pi, \downarrow}
\end{array}\right),
$$

where

$$
W=\left[\begin{array}{llll}
\varepsilon_{A B \uparrow}^{+}+\alpha_{11} & \alpha_{12} & \varepsilon_{A B \uparrow}^{-} & 0 \\
\alpha_{21} & \varepsilon_{A B \downarrow}^{+}+\alpha_{22} & 0 & \varepsilon_{A B \downarrow}^{-} \\
\varepsilon_{A B \uparrow}^{-} & 0 & \varepsilon_{A B \uparrow}^{+}-\alpha_{11} & -\alpha_{12} \\
0 & \varepsilon_{A B \downarrow}^{-} & -\alpha_{21} & \varepsilon_{A B \downarrow}^{+}-\alpha_{22}
\end{array}\right]
$$




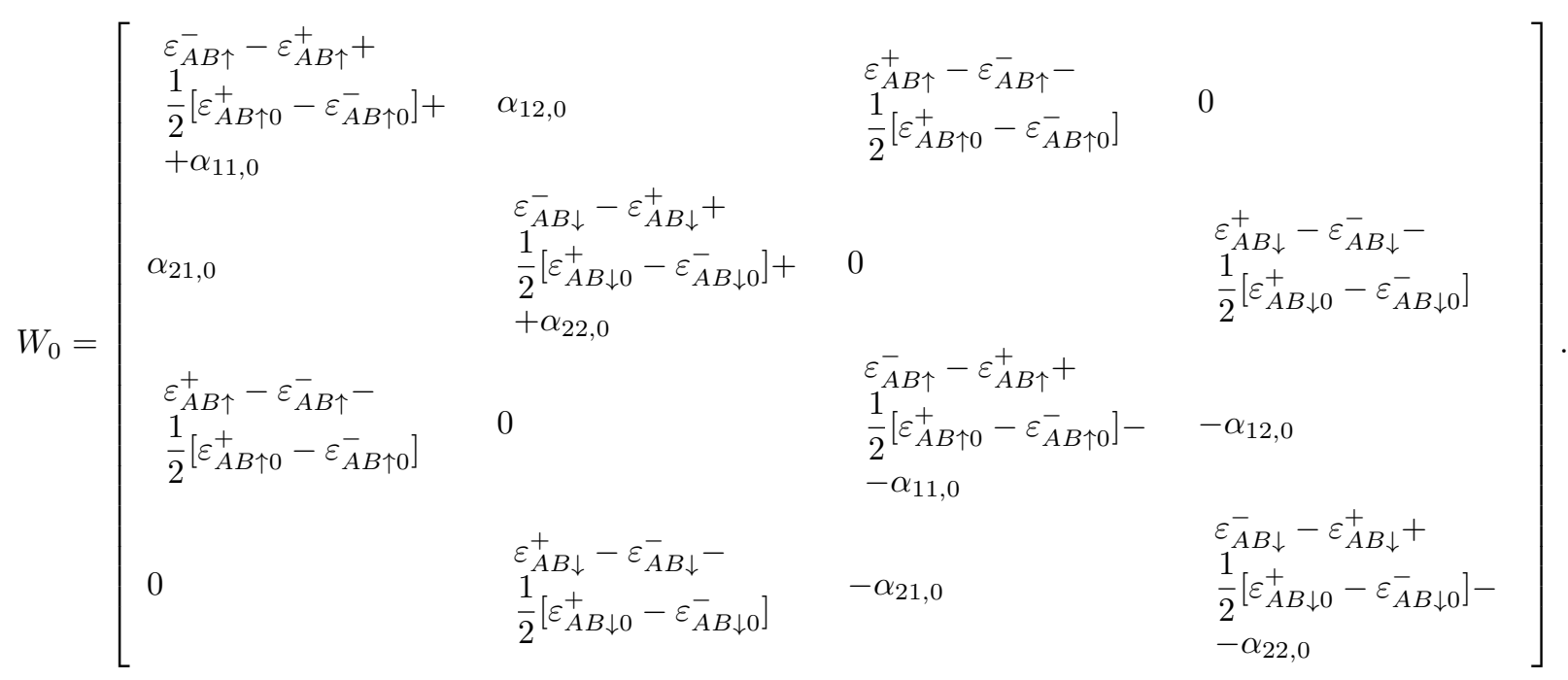

$H_{e}^{M}$ can be exactly diagonalized numerically to obtain the energies $E_{i}$ and distribution functions $f\left(E_{i}\right)$, where:

$$
f\left(E_{i}\right)=\frac{1}{\left[e^{\beta\left(E_{i}-\mu\right)+1}\right]}
$$

The ground state energy can be finally written as:

$$
E_{G S}=\sum_{i} \frac{2 N-1}{2 N} E_{i} f\left(E_{i}\right)+\sum_{i} \frac{1}{2 N} E_{i} f\left(E_{i}\right)+2 K_{1}+K_{0} .
$$

The persistent current and the Drude weight can be evaluated from the following relations:

$$
\begin{gathered}
I_{p c}=-\frac{1}{2 \pi}\left(\frac{\partial E_{G S}}{\partial \Phi}\right), \\
D W=\frac{N}{4 \pi^{2}} \frac{\partial^{2} E_{G S}}{\partial \Phi^{2}}, \quad \Phi=\Phi_{m},
\end{gathered}
$$

where $\Phi_{m}$ is the location minimum of $E_{G S}$ and can take values 0 or $1 / 2$ depending on the parity of the number of electrons.

\section{Numerical results}

We set $t=1, K_{B} T=0.1, U=0, g_{1}=g_{2}=0$ and measure all energies in units $\hbar \omega_{0}$ (see Fig. 2), the persistent current (see Fig. 3) and the Drude weight (see Fig. 5).

In Fig. 2 the dependence of the ground state energy $E_{G S}$ on the flux $\Phi$ is represented for different values of Rashba spin-orbit constant $\alpha$. One can see that the ground state energy is periodic with $\Phi$ and monotonically changes with $\alpha$.

In Fig. 3, we plot the persistent current $I_{p c}$ for different values of $\alpha$. The phase of the persistent current changes when $\alpha$ exceeds a critical value $\alpha_{c}$.

The variation of persistent current $I_{p c}$ as a function of $\alpha$ is represented on Fig. 4. When $\alpha>\alpha_{c}, I_{p c}$ with $\alpha$ becomes monotonically increasing.

The dependence of the Drude weight as a function of flux $\Phi$ for different $\alpha$ is shown in Fig. 5 .

The finite value of the DW indicates that the system is in the metallic phase. One can see that there is no periodicity and when $\alpha$ increasing, the minimum value varies greatly.

To compare these results to a single ring [6], they are similar in cases for $E_{G S}$ and $I_{p c}$ as a functions of the flux $\Phi$ for different $\alpha$ and differ considerably for the Drude weight function. The Drude weight as a function of flux $\Phi$ for different $\alpha$ in a single ring is a periodic function and the period changes with the change of $\alpha$.

Then, to understand the effect of electron-electron interaction, we set $t=1, K_{B} T=0.1, \alpha=2, g_{1}=g_{2}=0$ and measure the persistent current for different values of $U(U=0, U=1.4, U=1.8, U=2.2, U=2.4)$. The behavior of $I_{p c}$ as a function of $U$ with (when $\alpha=2$ ) and without (when $\alpha=0$ ) Rashba spin-orbit interaction is shown in Fig. 6. The behavior of $I_{p c}$ for such values of $U$ has a small difference.

The variation of persistent current for different values of $U$ with $\alpha=0,2$ is shown of Fig. 7. In absence of Rashba spin-orbit interaction when $\alpha=2$ there seem to exist a critical value $U_{c}$. When $U>U_{c}$ the persistent current decreases when $U$ increases. The decrease is quite rapid. Such affect appears as when $U$ increases the 


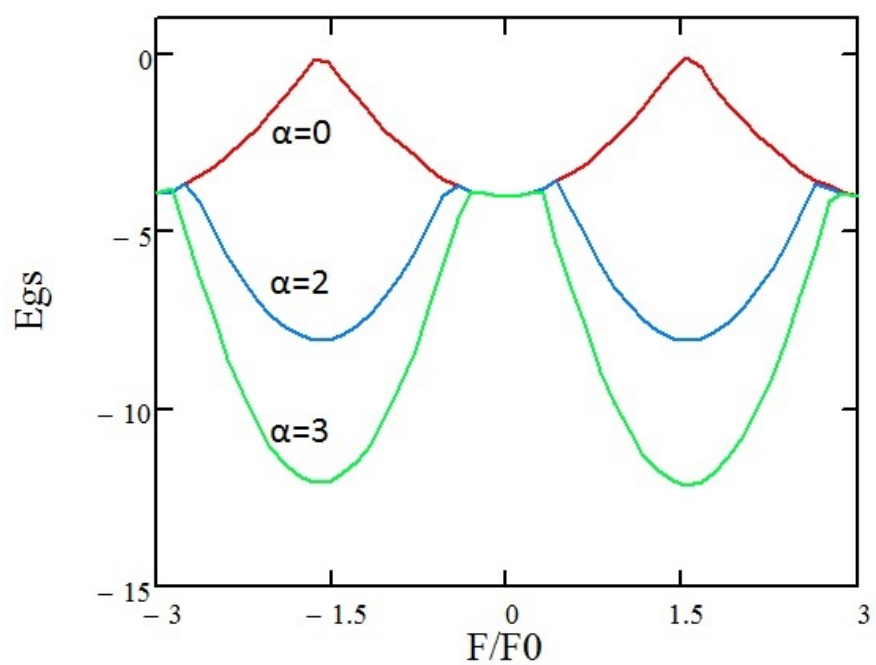

FIG. 2. The ground state energy $E_{G S}$ as a function of the flux $\Phi$ for different $\alpha$

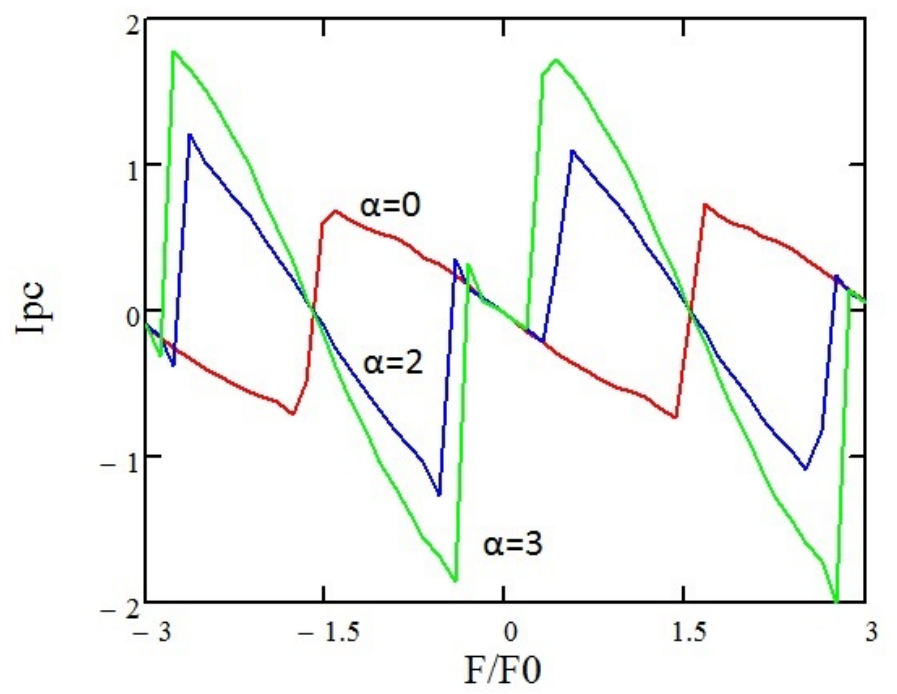

FIG. 3. The persistent current $I_{p c}$ as a function of the flux $\Phi$ for different $\alpha$

electrons experience a larger onsite repulsion and find it more difficult to hop from one site to another and this reduces the persistent current.

Next, we explore the effects of on-site electron-phonon interaction. We set $t=1, K_{B} T=0.1, \alpha=0$, $U=g_{2}=0$ and measure the persistent current (see Fig. 8) for different values of $g_{1}\left(g_{1}=0.1, g_{1}=0.5\right.$, $g_{1}=0.9$ ). The function for $g_{1}=0$ is similar to function $g_{1}=0.1$ with the difference that could be neglected. It is evident that persistent current decreases when $g_{1}$ increases. The increase of $g_{1}$ leads to deepening of the self-trapping polarization potential causing localization which will inhibit conduction.

The variation of persistent current for different values of $g_{1}$ with $\alpha=0, \alpha=2$ on Fig. 9 shows the effects of $g_{1}$ on persistent current with and without Rashba spin-orbit interaction. When there is no Rashba spin-orbit interaction, the $I_{p c}$ decreases as $g_{1}$ increases. The gradient of the curve vanishes monotonically. When there is a Rashba spin-orbit interaction $(\alpha=2)$ there seem to exist a critical value $g_{1 c}$. The $I_{p c}$ decreases when $g_{1}>g_{1 c}$ increases.

Next, to study the NN electron-phonon interaction, we set $t=1, K_{B} T=0.1, \alpha=0, U=g_{1}=0$ and measure the persistent current (see Fig. 10) for different values of $g_{2}\left(g_{2}=0.1, g_{2}=0.3, g_{2}=0.6\right)$. The function of $I_{p c}$ for $g_{2}=0$ is similar to function $g_{2}=0.1$ with the difference that could be neglected. The behavior of $g_{2}$ is similar for that of $g_{1}$ but the effect of $g_{2}$ on persistent current is stronger. The periodicity of $I_{p c}$ decreases when $g_{2}$ increases. 


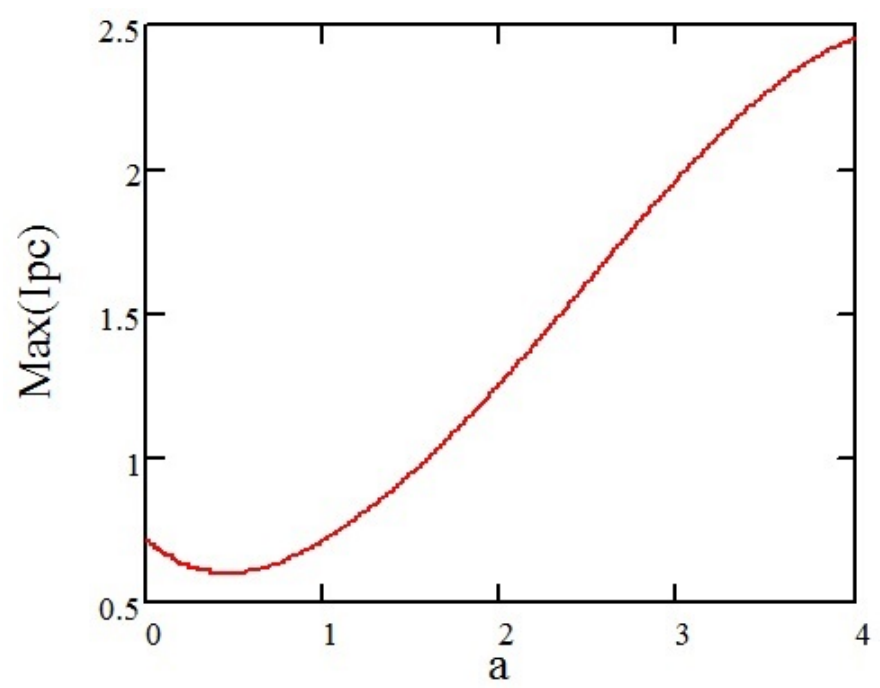

FIG. 4. Variation of $I_{p c}$ as a function of $\alpha$
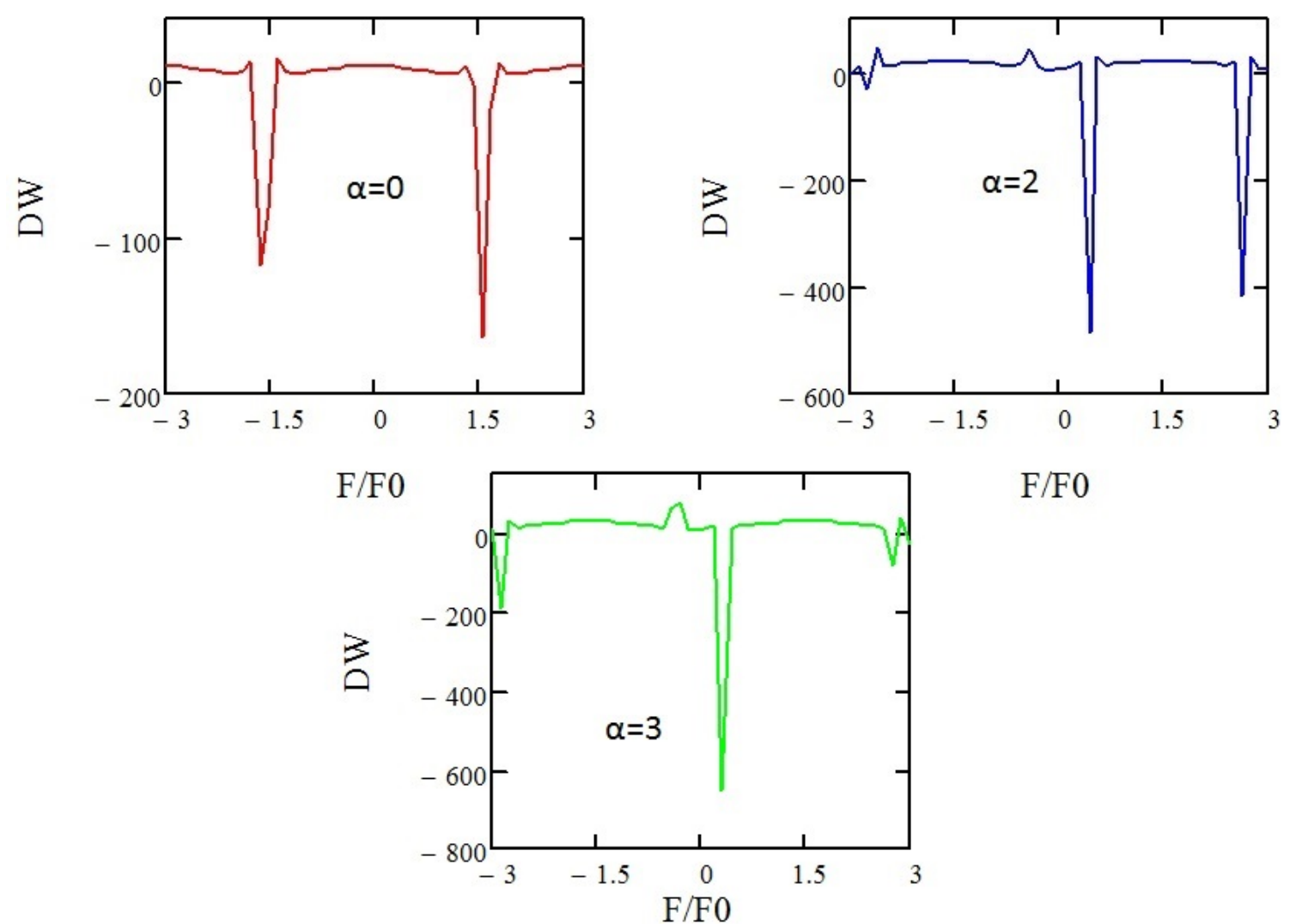

F/F0

FIG. 5. The Drude weight as a function of flux $\Phi$ for different $\alpha$ 

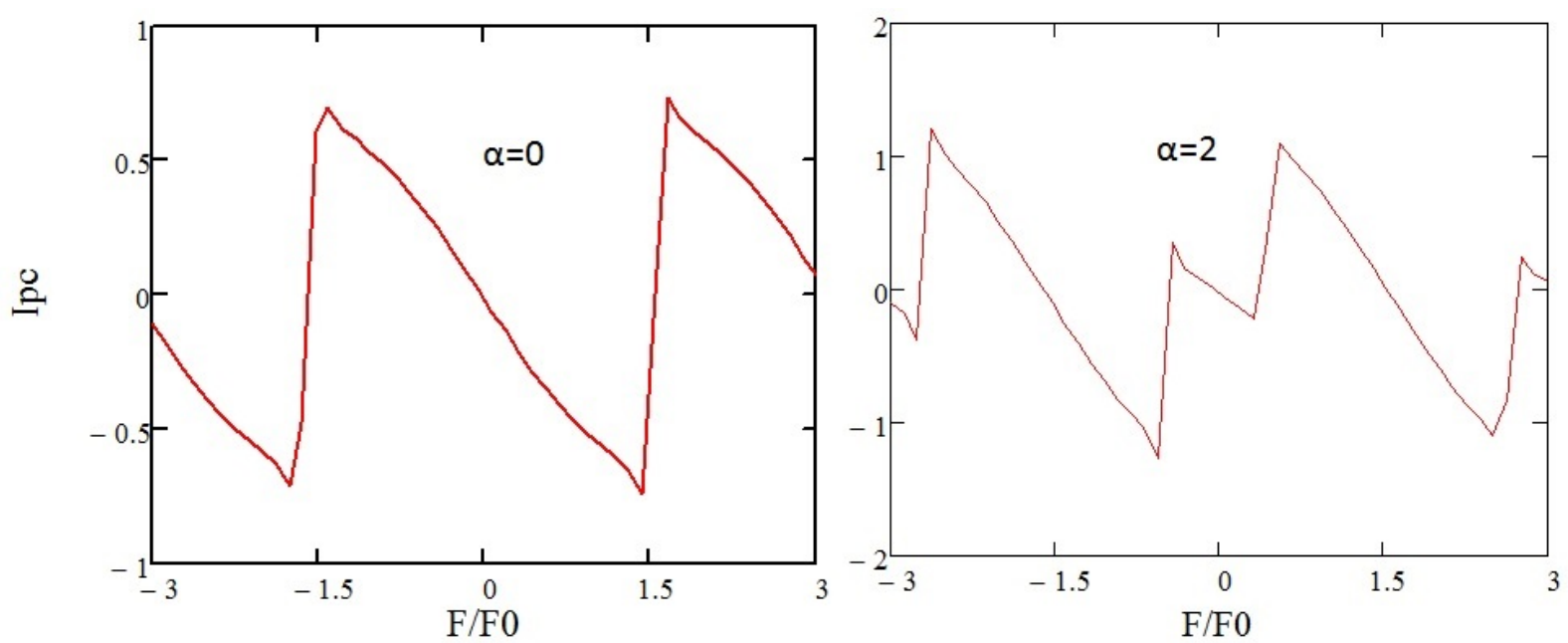

FiG. 6. Persistent current $I_{p c}$ for different values of $\mathrm{U}$ with $\alpha=0,2$
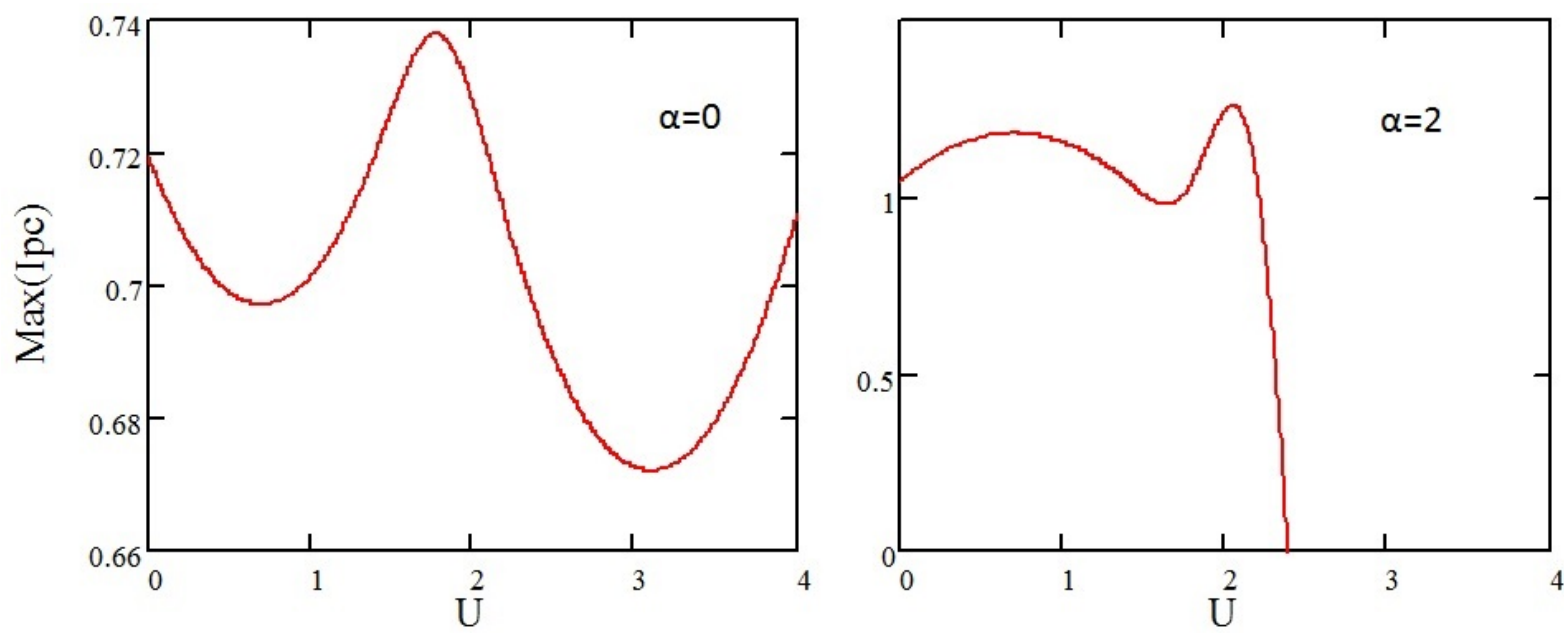

FIG. 7. Variation of persistent current $I_{p c}$ for different values of $U$ with $\alpha=0,2$

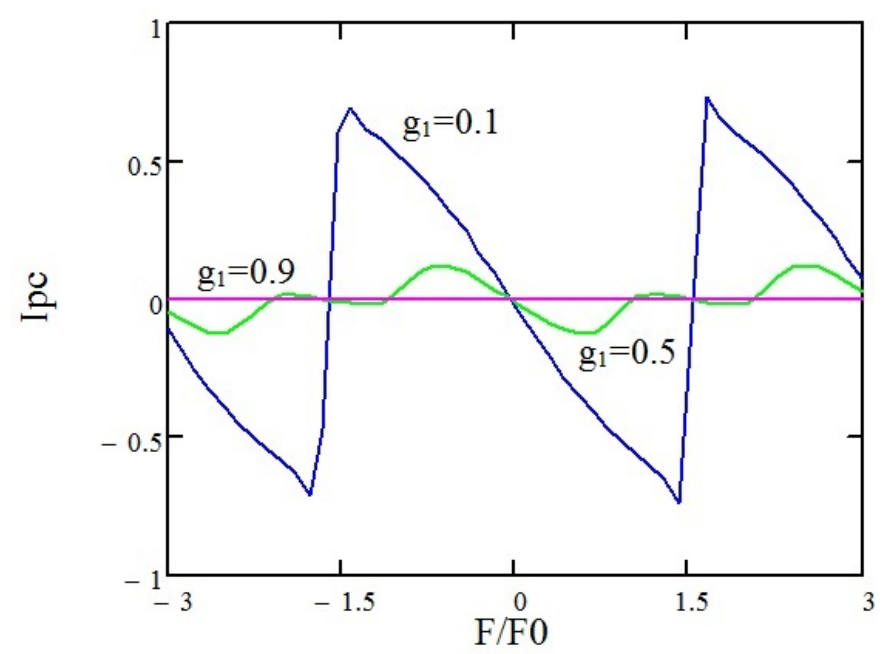

FIG. 8. Persistent current $I_{p c}$ for different values of $g_{1}$ with $\alpha=0$ 


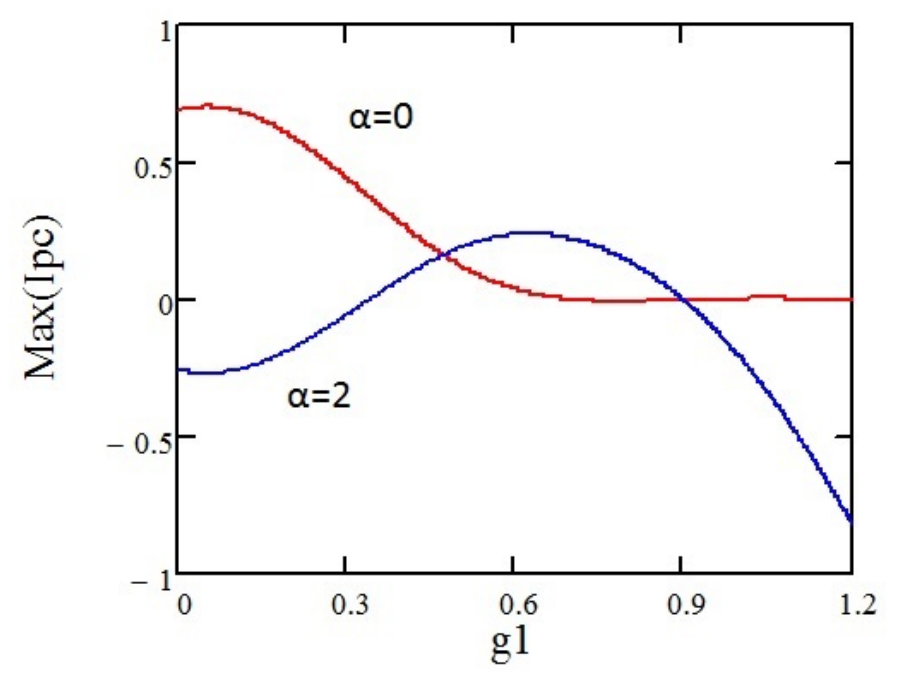

FIG. 9. Variation of persistent current $I_{p c}$ for different values of $g_{1}$ with $\alpha=0, \alpha=2$

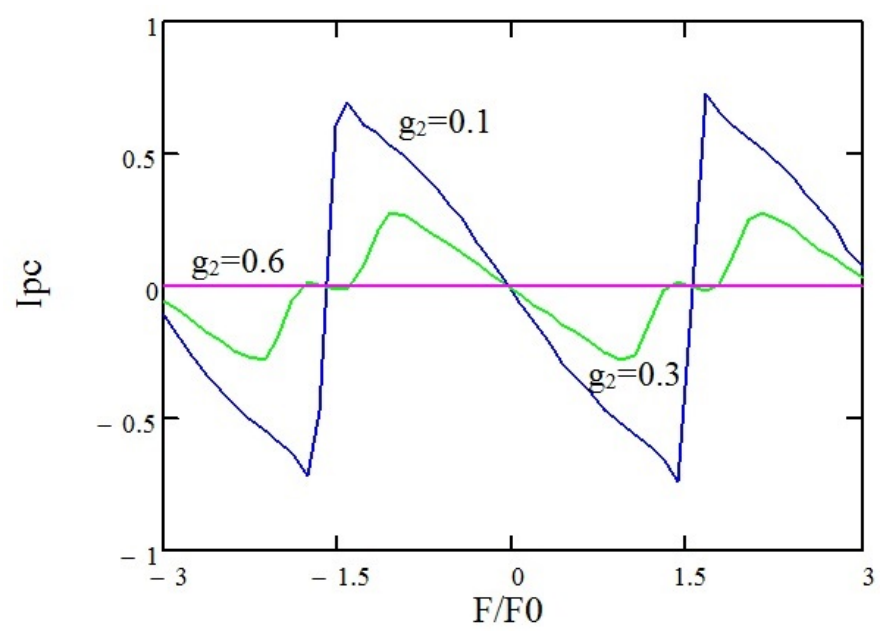

FIG. 10. Persistent current $I_{p c}$ for different values of $g_{2}$ with $\alpha=0$

In Fig. 11 we show the dependence of persistent current $I_{p c}$ on $g_{2}$ with $\alpha=2, g_{1}=0.9$ in the presence of on-site electron-phonon interaction. The periodicity of $I_{p c}$ also decreases.

The next important step is to study the effects of temperature on persistent current. We set $t=1, \alpha=0$, $U=0, g_{1}=g_{2}=0$ and measure the persistent current (see Fig. 12) for different values of $K_{B} T\left(K_{B} T=0.1\right.$, $K_{B} T=0.24, g_{2}=0.46, K_{B} T=0.71$ ) and for $\alpha=2$ (see Fig. 13).

Both Fig. 12,13 show that persistent current decreases as temperature increases. According the Fig. 14, there appears to exist a critical value $\left(k_{B} T\right)_{c}$, and when $k_{B} T>\left(k_{B} T\right)_{c}$, the persistent current decreases.

In the presence of Rasba spin-orbit interaction we plot the variation of persistent current $I_{p c}$ for different values of $k_{B} T$ with $\alpha=2, g_{1}=0.5$ and compare it with the variation for $\alpha=2, g_{1}=0$. The persistent current also decreases when temperature increases (Fig. 15).

Finally, we will study the effect of chemical potential on persistent current. On Fig. 16 the dependence of persistent current on $\Phi$ for different values of chemical potential $\mu$ is shown. We set $t=1, K_{B} T=0.1, \alpha=0$, $U=0, g_{1}=g_{2}=0$ and measure the persistent current for different values of $\mu(\mu=0, \mu=0.01, \mu=0.03$, $\mu=0.05)$ and $\alpha=2$. One can see that the resulted figures are quite similar. So the changes of magnitude and phase of persistent current with $\mu$ are small. This result is different from what is presented in [6] for similar quantum ring. 


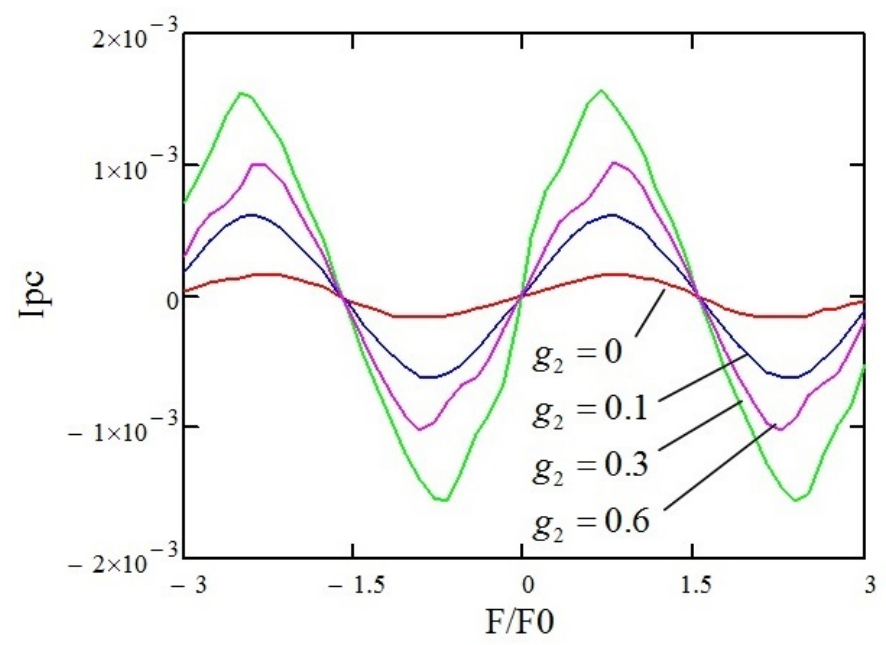

FIG. 11. Persistent current $I_{p c}$ for different values of $g_{2}$ with $\alpha=2, g_{1}=0.9$

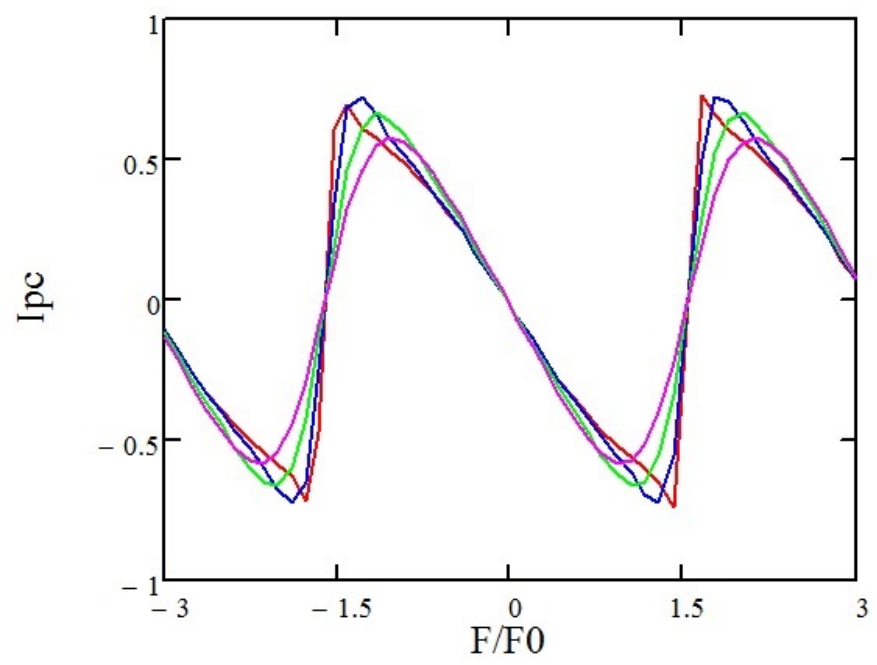

FIG. 12. Persistent current $I_{p c}$ for different values of $k_{B} T$ with $\alpha=0$

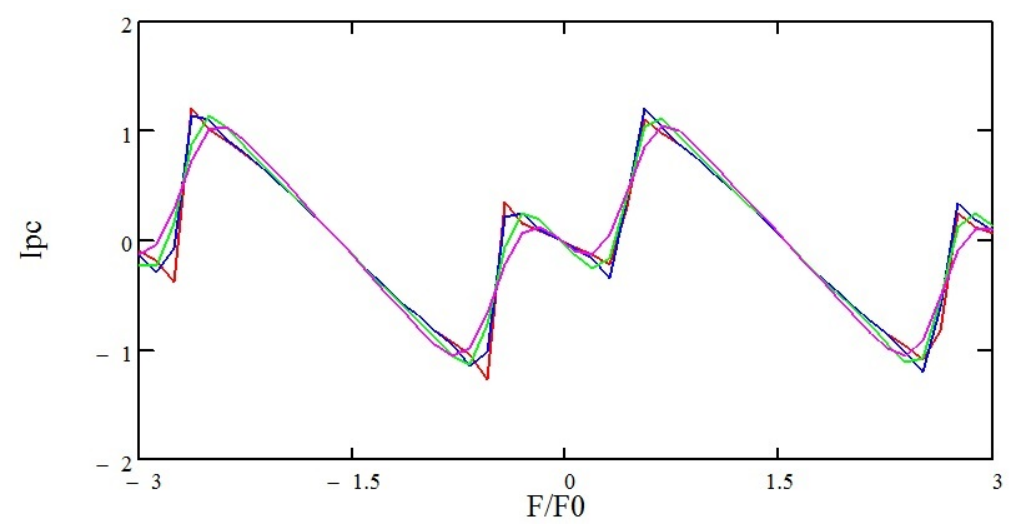

FIG. 13. Persistent current $I_{p c}$ for different values of $k_{B} T$ with $\alpha=2$ 


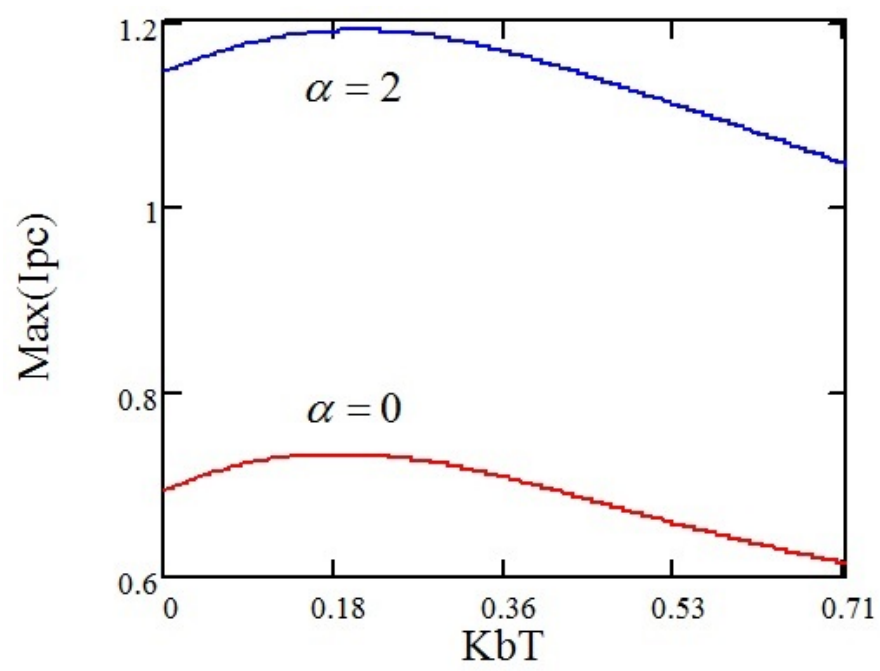

FIG. 14. Variation of persistent current $I_{p c}$ for different values of $k_{B} T$ with $\alpha=0,2$
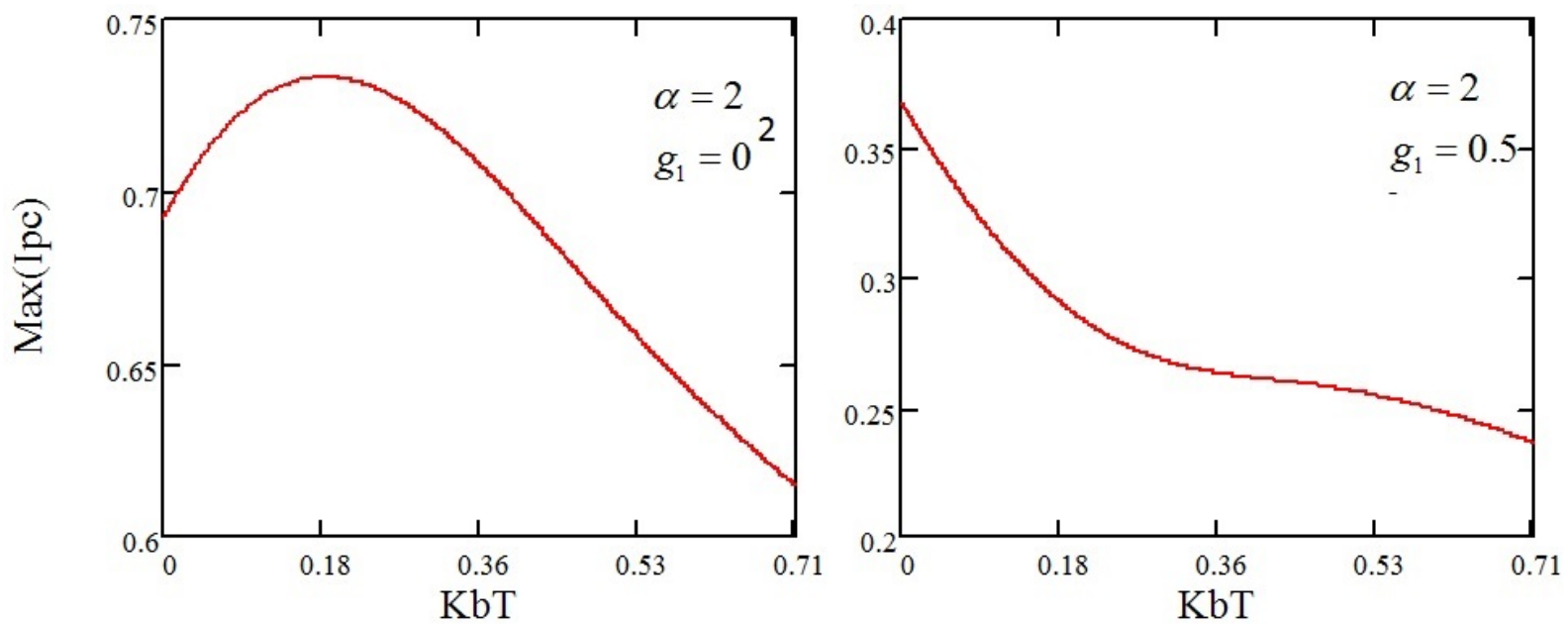

FIG. 15. Variation of persistent current $I_{p c}$ for different values of $k_{B} T$ with $\alpha=2, g_{1}=0$, $\alpha=2, g_{1}=0.5$

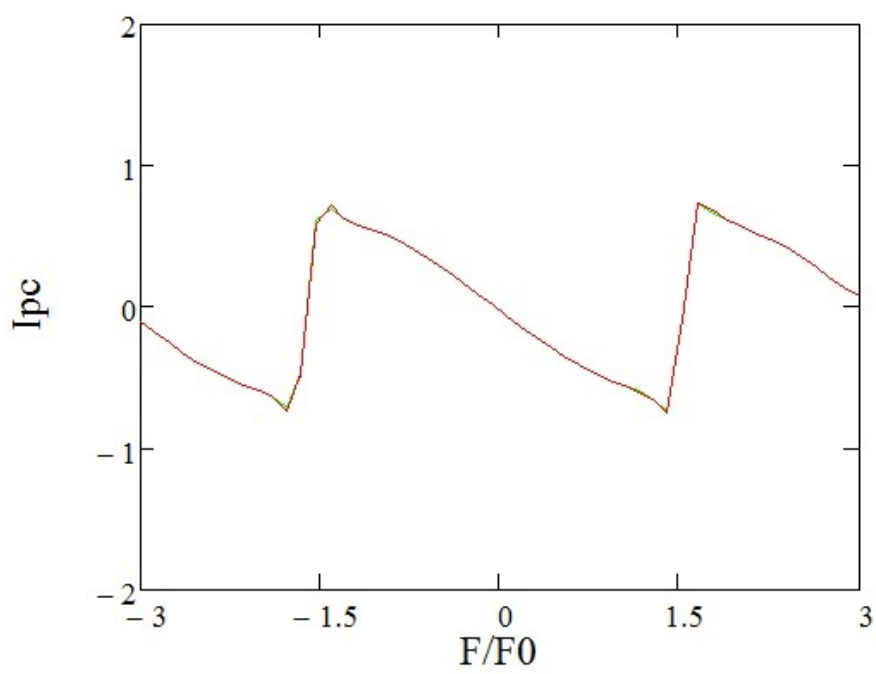

FIG. 16. Persistent current $I_{p c}$ as a function of flux $\Phi$ for different values of $\mu, \alpha=0$ 
The variation of persistent current $I_{p c}$ for different values of $\mu$ in the presence $(\alpha=2)$ and absence $(\alpha=0)$ of Rasba spin-orbit interaction is shown in Fig. 17. In both cases there seem to exist a critical value $\mu_{c}$ such that when $\mu>\mu_{c}$ the persistent current decreases.

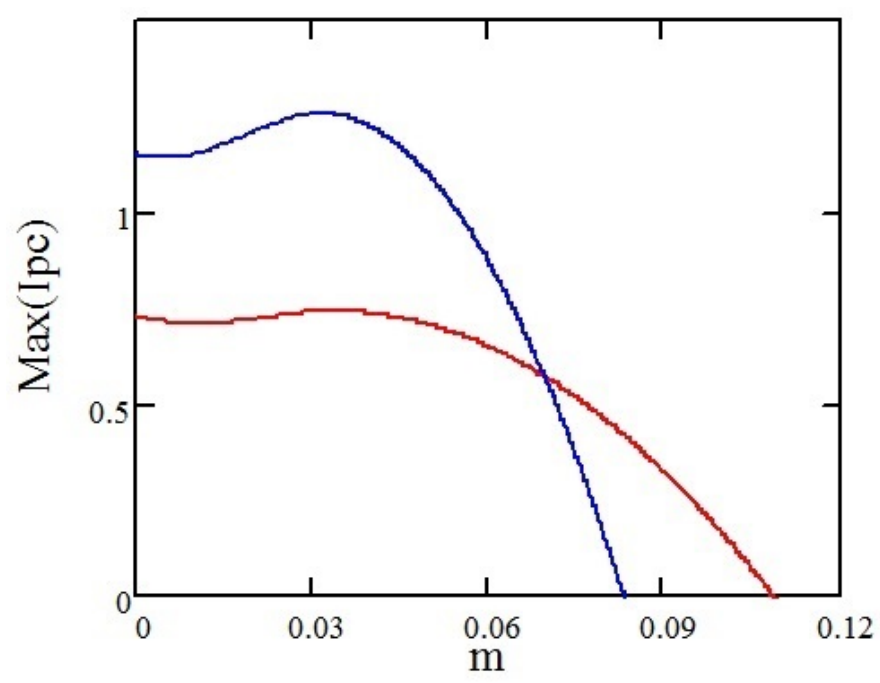

FIG. 17. Variation of persistent current $I_{p c}$ for different values of $\mu, \alpha=0,2$

\section{Conclusions}

Thus during the work, the model of two chained Holstein-Hubbard rings in the presence of Rashba spin-orbit interaction and the effect of Rashba spin-orbit interaction were obtained. First, the phonons degrees of freedom were eliminated by the conventional Lang-Firsov transformation and then the spin-dependence was removed by performing the unitary transformation, the effective electronic Hamiltonian was diagonalized by using the HartreeFock approximation. The equations for ground state energy, persistent current and Drude weight were also obtained. The persistent current was calculated by differentiating the GS energy. The dependence of ground state energy, persistent current and Drude weight as a functions of flux for different values of Rashba spin-orbit interaction was numerically shown. Next, the effects of Aharonov-Bohm flux, temperature, chemical potential spin-orbit interaction and electron-phonon interaction on the persistent current were also investigated.

\section{Acknowledgements}

This work was partially financially supported by the Government of the Russian Federation (grant 08-08), by grant 16-11-10330 of Russian Science Foundation. The authors thank Prof. R. Figari and Prof. W. Florek for interesting discussion.

\section{References}

[1] Buttiker M., Imry Y. and Landauer R. Josephson behavior in small normal one-dimensional rings. Phys. Lett. A, 1983, 96, P. $365-267$.

[2] Cheung H.-F., Gefen Y., Riedel E.K. and Shih W.-H. Persistent current in small one-dimensional metal rings. Phys. Rev. B, 1988, 37, P. 6050-6062

[3] Takada Y. and Chatterjee A. Possibility of a metallic phase in the charge-density-wave-spin-density-wave crossover region in the onedimensional Hubbard-Holstein model at half filling. Phys. Rev. B., 2003, 67, P. 081102.

[4] Sil S., Maiti S.K. and Chakrabarti A. Interplay of magnetic field and geometry in magneto-transport of mesoscopic loops with Rashba and Dresselhaus spin-orbit interactions. J. Appl. Phys., 2012, 112, P. 024321.

[5] Manolescu A.,Daday C.,Gudmundsson V. Coulomb effects on the spin polarization of quantum ring. Nanosystems Phys. Chem. Math., 2011, 2(3), P. 29-36.

[6] Monisha P.J., Sankar I.V., Sil S. and Chatterjee A. Persistent current in a correlated quantum ring with electron-phonon interaction in the presence of Rashba interaction and Aharonov-Bohm flux. Scientific Reports, 2016, 6, P. 20056.

[7] Voda A. Micro, Nanosystems and Systems on Chips: Modeling, Control, and Estimation. New York, John Wiley \& Sons, 2013.

[8] Eremin D.A., Grishanov E.N., Nikiforov D.S., Popov I.Y. Wave dynamics on time-depending graph with Aharonov-Bohm ring. Nanosystems: Physics, Chemistry, Mathematics, 2018, 9(4), P. 457-463.

[9] Lang I. and Firsov Y.A. Kinetic theory of semiconductors with low mobility. Sov. Phys. JETP, 1963, 16(5), P. 1301-1312.

[10] Cabib D. and Callen E. Charge order and antiferromagnetic order in the Hubbard model with nearest neighbor Coulomb interaction: Weak coupling. Phys. Rev. B, 1975, 12, P. 5249-5254. 